\title{
Total improvement after surgery for obstructive sleep apnea syndrome in a patient with concurrent malignant idiopathic intracranial hypertension
}

\author{
Halil Onder, MD, ${ }^{1}$ Onur Ergun, MD, ${ }^{2}$ Mehmet Kaygisiz, MD, ${ }^{3}$ and I. Samet Daltaban, MD ${ }^{4}$ \\ Departments of ${ }^{1}$ Neurology, ${ }^{2}$ Otolaryngology, and ${ }^{3}$ Ophthalmology, Yozgat State Hospital; and ${ }^{4}$ Department of Neurosurgery, \\ Bozok University Hospital, Yozgat, Turkey

\begin{abstract}
The association between idiopathic intracranial hypertension $(\mathrm{IIH})$ and obstructive sleep apnea syndrome (OSAS) constitutes an interesting point of discussion regarding clinical approaches as well as the underlying pathophysiological mechanisms. Here, the case of a 42-year-old female with malignant IIH and OSAS is illustrated. Initially, the patient was treated with lumboperitoneal shunt surgery. However, OSAS surgery provided total recovery from the recurrent symptoms developing secondary to lumboperitoneal shunt malfunction. The authors point out the importance of identifying OSAS as a crucial causative agent in some IIH subtypes. Future studies are warranted to clarify this underestimated association.
\end{abstract}

https://thejns.org/doi/abs/10.3171/2018.3.JNS171663

KEYWORDS idiopathic intracranial hypertension; obstructive sleep apnea; malignant course; papilledema; pathophysiology; hydrocephalus

I DIOPATHIC intracranial hypertension (IIH) is a syndrome of unknown etiology, with patients presenting with headache, papilledema, and vision loss. Generally, the clinical course of $\mathrm{IIH}$ is favorable and responsive to medical treatment. Very occasionally, however, severe visual loss can be present in the acute phase, defined as "malignant IIH," which can lead to permanent visual loss and requires prompt, intensive treatment interventions. ${ }^{12}$ In this context, an interesting topic of discussion is the possible relationship between $\mathrm{IIH}$ and obstructive sleep apnea syndrome (OSAS). ${ }^{17}$ Although the association between these entities has been mentioned previously, ${ }^{17,18}$ there is no consensus at this point given the conflicting results of these studies. Remarkably, in a unique pediatric case, IIH symptoms improved in a patient with IIH following OSAS surgery. ${ }^{9}$ Herein, we present an adult patient with malignant IIH who had been unsuccessfully treated with lumboperitoneal shunt surgery. Recurrent symptoms and progressive visual loss due to lumboperitoneal shunt malfunction were totally cured by OSAS surgery in the follow-up. In a detailed illustration of this rare case, we discuss OSAS as a causative agent in IIH, particularly in the malignant subtypes with progressive papilledema.

\section{Case Report}

History and Examination

A 42-year-old female with complaints of headache and bilateral impaired vision for the last 3 months was admitted to our institution. The patient described her headaches as bilateral, frontal, and pressure-like, which worsened while supine. She had blurry vision in all four quadrants and bilateral "blobs" in her visual field. She had no medical history except a body mass index of $35 \mathrm{~kg} / \mathrm{m}^{2}$ (height: $160 \mathrm{~cm}$, weight: $90 \mathrm{~kg}$ ). On neurological examination, Frisén stage II papilledema was assessed and visual acuity was normal bilaterally. ${ }^{6}$ Cranial MRI and MR venography showed normal findings. With a provisional diagnosis of IIH, lumbar puncture (LP) was conducted. Opening pressure was measured as $27 \mathrm{~cm} \mathrm{H}_{2} \mathrm{O}$, and $25 \mathrm{ml}$ of CSF was drained, resulting in significant improvement in the patient's headaches and vision disturbance. Hence, the diagnosis of IIH was established, and after a 7-day in-hospital monitoring period, the patient was discharged on $250 \mathrm{mg}$ of acetazolamide three times a day and $25 \mathrm{mg}$ of topiramate two times a day with a plan for outpatient follow-up.

At a follow-up visit 2 weeks later, however, the patient

ABBREVIATIONS CC-MMA = counterclockwise maxillomandibular advancement; CPAP = continuous positive airway pressure; EES = Epworth Sleepiness Scale; ICP = intracranial pressure; IIH = idiopathic intracranial hypertension; $\mathrm{LP}=$ lumbar puncture; $\mathrm{OCT}$ = optical coherence tomography; OSAS = obstructive sleep apnea syndrome. SUBMITTED July 7, 2017. ACCEPTED March 19, 2018.

INCLUDE WHEN CITING Published online August 17, 2018; DOI: 10.3171/2018.3.JNS171663. 


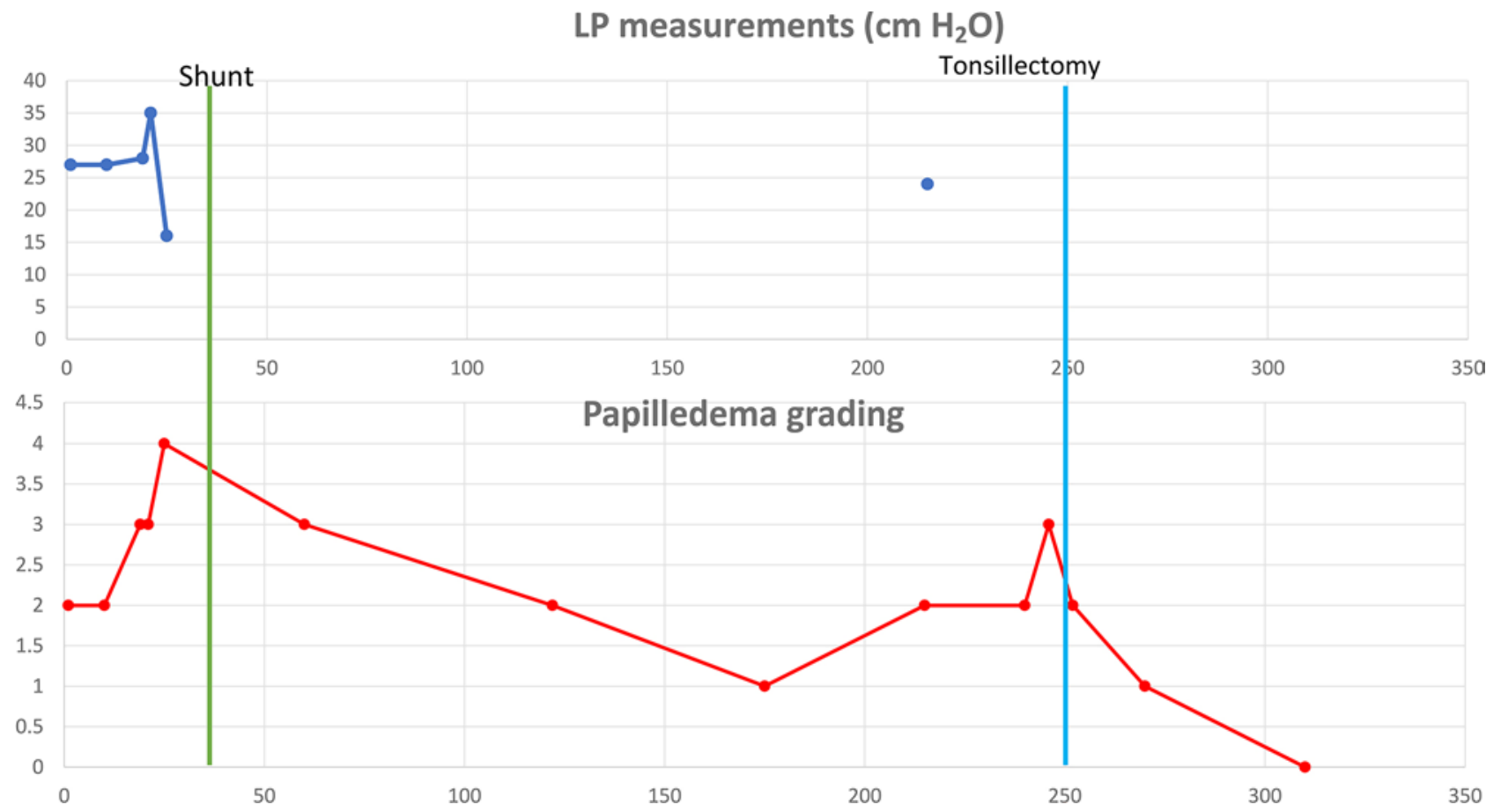

FIG. 1. Graphic representation of the temporal evolution of LP opening pressures and papilledema grading during the patient's clinical course. Green line indicates the time of lumboperitoneal shunt surgery; light blue line, the time of OSAS surgery; dark blue line and dot, the LP measurements; and red line, the papilledema stage. The x-axis represents the number of days. Figure is available in color online only.

reported suffering from headache progression and visual disturbances. Fundus examination showed worsening of the papilledema (Frisén stage III), and visual acuity was 6/9 bilaterally. The patient was hospitalized for the second time, and a second LP revealed an opening pressure of $27 \mathrm{~cm} \mathrm{H}_{2} \mathrm{O}$. Thirty milliliters of CSF was drained, and medications were gradually increased to $250 \mathrm{mg}$ of acetazolamide five times a day and $50 \mathrm{mg}$ of topiramate twice a day. Nevertheless, no recovery was achieved in the following 10-day hospitalization period. Instead, her symptoms progressed, and her visual acuity deteriorated to the level of $4 / 5$ bilaterally. Fundus examination at that time revealed newly emerging papillary hemorrhages. During that interval, LP treatment was administered two more times with opening pressures of 28 and $35 \mathrm{~mm} \mathrm{H}_{2} \mathrm{O}$, respectively, which failed to help the patient's clinical status. Given the malignant clinical course of the patient, neurosurgery was consulted urgently, and lumboperitoneal shunt surgery was decided on. Following shunt surgery, a slight recovery was achieved in the acute period. Therefore, to be sure that the shunt was working, LP was repeated in the 1st week after the shunt surgery, which showed normalization of the CSF pressure (opening pressure 16 $\mathrm{mm} \mathrm{H}_{2} \mathrm{O}$ ). For the second time, the patient was discharged on intensive medical therapy ( $250 \mathrm{mg}$ of acetazolamide five times a day, $50 \mathrm{mg}$ of topiramate twice a day). In the subacute period, a moderate recovery was achieved.

Eight months later, the patient was admitted with worsening headaches and visual disturbances over the last month. Given the progression of her papilledema, she was hospitalized and LP was performed, which showed an opening pressure of $24 \mathrm{~mm} \mathrm{H}_{2} \mathrm{O}$, proving malfunction of the lumboperitoneal shunt (Fig. 1).

When the patient was further evaluated, OSAS was suspected. Thus, otolaryngology was consulted. Otolaryngological examination revealed significantly hyponasal speech, a history of severe witnessed apneas, and prominent morning symptoms, such as sleeplessness and irritability, together with severe headaches. The patient also complained about dyspnea and unbearable worsening of sleep quality during upper respiratory tract infections. Physical examination showed grade 4 tonsillar hypertrophy (occupying more than $75 \%$ of the airway), class 2 oropharynx, and grade 2 hypopharynx. Taken together, these findings indicated tonsillectomy due to obstructive tonsillar hypertrophy. Preoperative polysomnography was not needed. The Epworth Sleepiness Scale (ESS) score was calculated as 17. As a result of interdepartmental consultations and recent literature data pointing to an association between OSAS and IIH in terms of a causal relationship, ${ }^{18}$ surgery was predicted to improve IIH symptoms as well as the OSAS symptoms, and shunt revision surgery was not considered in the acute period.

\section{Operation and Postoperative Course}

Tonsillectomy was performed successfully. Soon after the operation, significant amelioration of the OSAS symptoms was attained. Moreover, a dramatic recovery in IIH symptoms also occurred. Soon after surgery, she fully recovered from the headaches and visual symptoms, and 


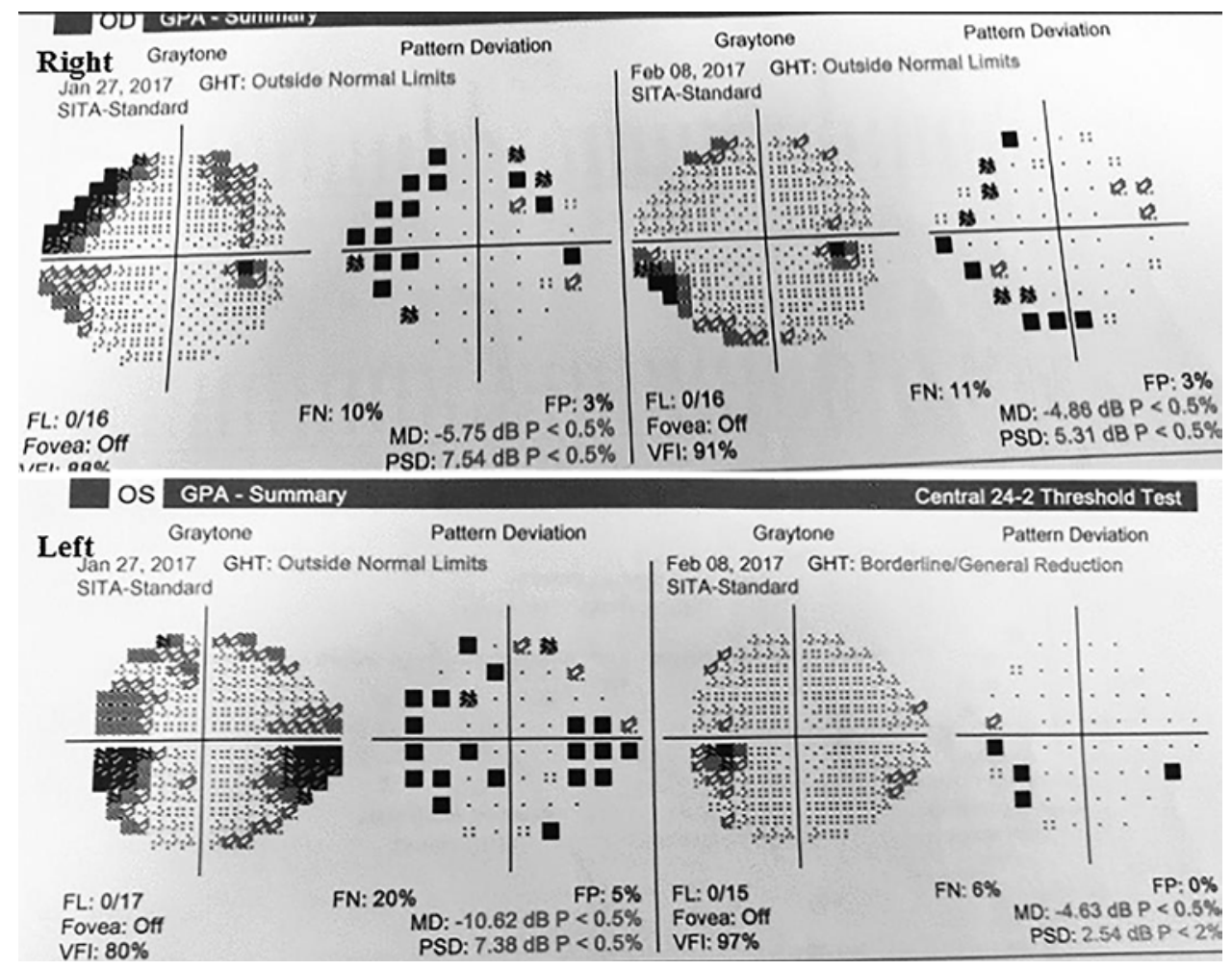

FIG. 2. Perimetry tests performed preoperatively and postoperatively (8th day) showing near-total recovery from the bilateral visual field defects, which occurred in the form of global constriction. Upper: Right eye. Lower: Left eye.

fundus examinations revealed the normalization of papilledema. Optical coherence tomography (OCT) also confirmed the gradual improvement of papilledema in comparison to its preoperative status, and perimetry showed total recovery of preoperative bilateral visual field defects (Figs. 2 and 3). By the last visit 3 months after surgery, the headaches had stopped and the papilledema had regressed to the level of bilateral stage I despite significant decrements in the medications (only $250 \mathrm{mg}$ of acetazolamide twice a day was continued). However, polysomnography, also performed at the 3rd postoperative month, revealed persistent OSAS but at a mild degree (Apnea Hypopnea Index: 14.6/hr). Remarkably, a concurrent ESS score was 0.

\section{Discussion}

Idiopathic intracranial hypertension is identified as benign intracranial hypertension because of its usual course of spontaneous resolution, leaving few sequelae. ${ }^{10}$ Our patient had developed progressive visual loss and worsening papilledema, which did not respond to LPs and high-dosage medications, thus confirming a diagnosis of malignant IIH. Although there is no prospective controlled study comparing conservative and surgical treatments, progressive visual loss not responding to repeated LPs and medical therapy is usually treated with surgery. ${ }^{2}$ In our case, prompt lumboperitoneal shunt insertion provided moderate improvement in resistant symptoms and gradual improvement in the papilledema. Eight months later, however, symptoms recurred and a newly performed
LP showed increased CSF pressure $\left(24 \mathrm{~mm} \mathrm{H}_{2} \mathrm{O}\right)$, confirming malfunction of the lumboperitoneal shunt. At that time, tonsillectomy was performed, which provided excellent improvement in the patient's symptoms (namely headache and visual complaints) as well as abnormal visual findings. In our opinion, this report of recovery from IIH via OSAS surgery may provide crucial insight into the mechanisms underlying IIH.

Obstructive sleep apnea syndrome can be described as a disorder characterized by repetitive episodes of hypopnea or apnea due to the collapse of the upper respiratory tract during sleep despite ongoing breathing effort. ${ }^{3}$ Polysomnography is a recommended tool for evaluating OSAS; however, there is no gold standard for the diagnosis. The association of OSAS and cerebral vasodilatation-linked intracranial pressure (ICP) increments is a recognized entity among specialists. ${ }^{8,15}$ Remarkable studies of ICP monitoring during sleep in patients with OSAS have shown normal ICP values during the awake period but a slow increase and then a steep increase in ICP during sleep. Given the findings in these studies, their authors have suggested that the slow increase may be associated with hypercapnia and hypoxia, whereas the steep increase at the end of apneic episodes has been related to an increase in intraarterial pressure and central venous pressure. ${ }^{8}$ On the other hand, central obesity has also been hypothesized to be an important factor for the development of IIH in OSAS, causing increased abdominal pressure, which leads to poor venous drainage from the brain, resulting in increased ICP. ${ }^{4}$ In a distinct report by Wardly et 


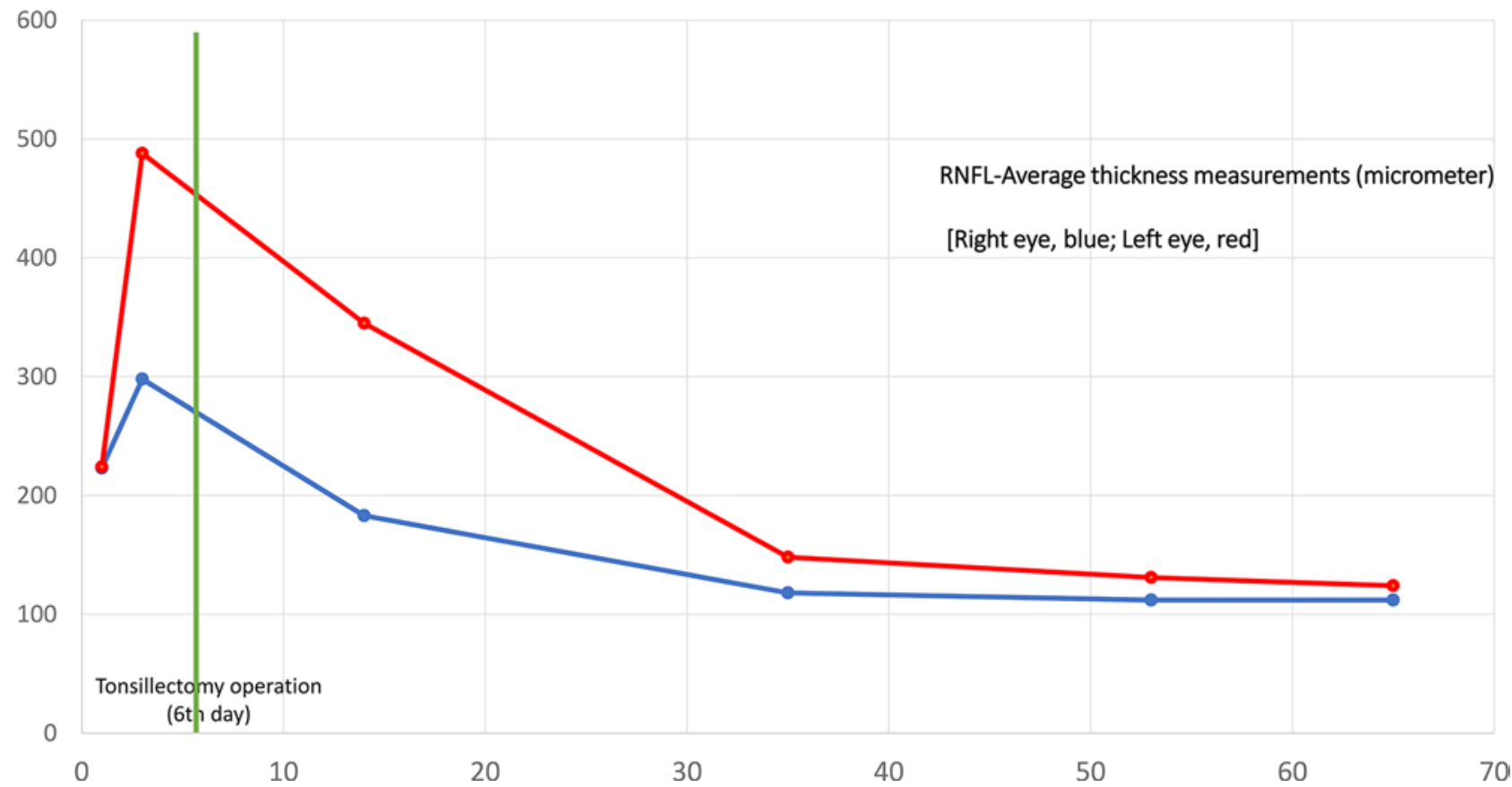

FIG. 3. Graphic representation of retinal nerve fiber layer (RNFL) measurements revealing gradual decrements in both the right eye and the left eye soon after the tonsillectomy operation. The $x$-axis represents the number of days and the $y$-axis the average thickness measurements in micrometers. Figure is available in color online only.

al., a view of upper airway structure compression in central obesity and its effect as a contributor to OSAS mechanisms has been discussed in the setting of IIH pathophysiology. ${ }^{17}$ In addition, Wardly has hypothesized a possible effect of obesity leading to a propensity for jugular venous collapsibility or narrowing, contributing to increased ICP via increased resistance in the jugular veins. ${ }^{18}$ Despite these hypotheses, however, there is still no consensus on an association between these two entities. For example, in a crucial polysomnography study conducted on 24 newly diagnosed IIH patients, OSAS was not found to be an independent factor in the severity and prevalence of IIH. ${ }^{16} \mathrm{In}$ accordance, papilledema was also not found in anyone in a large cohort of OSAS patients, leading to a reinvestigation of the influence of OSAS in increased ICP as well as its impact on IIH pathophysiology. ${ }^{14}$

Altogether, although the association between IIH and OSAS is a recognized issue of discussion, underlying mechanisms as well as a possible causality between these disorders have not been elucidated as yet. Hence, we think that our report may provide crucial insight into the impact of OSAS in IIH pathophysiology. Dramatic and rapid improvement (in both clinical and laboratory findings) after OSAS surgery was documented in our patient, strongly supporting the influence of OSAS as an etiological agent in IIH. For the treatment of OSAS, continuous positive airway pressure (CPAP) is recommended as first-line therapy, and surgical options should be considered when noninvasive medical therapy fails. ${ }^{3}$ In OSAS, various types of surgical modalities are indicated to modify the various sites of airway obstruction, including nasal surgery, surgeries of the oropharynx (tonsillectomy, etc.), and cra- niofacial surgery. ${ }^{5}$ In our patient, however, the obstructive tonsillar hypertrophy was prominent and thus we decided on tonsillectomy as the first-line therapy for OSAS.

In a previous report, gradual improvement of papilledema via CPAP treatment in a patient with OSAS was also documented. 7 In another unique report, a child who presented with IIH also had a complete and immediate cure via OSAS surgery. ${ }^{9}$

In an adult patient with IIH and accompanying OSAS, Wardly et al. found that counterclockwise maxillomandibular advancement (CC-MMA), a surgical modality for OSAS, provided recovery from IIH. ${ }^{17}$ Nevertheless, these authors drew attention to the persistence of OSAS and formed their hypothesis on the basis of a mechanism that could be solely associated with specific features of CC-MMA, rather than OSAS pathophysiology. Hence, they suggested that the positive outcome was effected via mandibular advancement producing a decrease in jugular venous resistance, allowing improved absorption of CSF. In this patient, however, and as discussed by the authors, there may be some crucial questions regarding the actual existence of $\mathrm{IIH}$, as papilledema was absent and a prominent increase in ICP had not occurred. Although residual findings of OSAS symptoms persisted in the follow-up of the patient, a significant recovery was achieved, which seems to be underestimated in the report. Hence, we think that the rationale for focusing on a mechanism of jugular venous resistance decrease, rather than on recovering from OSAS pathophysiology (which is a much more supported theory), may also be questionable. To our knowledge, ours is the first report of an adult IIH patient successfully treated with tonsillectomy by aiming to re- 
lieve OSAS. The rapid recovery was also demonstrated via serial OCT tests, a useful quantitative tool for the determination and follow-up of papilledema throughout its whole clinical course.,13 Through the illustration of our extremely rare case and the related literature review, we have clearly shown OSAS as an independent etiological factor in IIH, which should be considered, particularly in patients with malignant IIH. An interesting point in our patient was the prominent finding of papilledema, which did not have an overwhelming response to shunt surgery (even in the immediate postoperative period). The absence of a response may be explained by mechanisms other than ICP and by the OSAS itself. For instance, OSAS-related increased oxidative stress and inflammation, which lead to neuronal damage and apoptosis, have been hypothesized to be associated with optic nerve pathology. ${ }^{7}$ Of note, papilledema has also been frequently reported in OSAS patients who did not meet the criteria for IIH, ${ }^{7,11}$ Future studies with a larger number of patients with both IIH and OSAS are warranted to clarify these arguments. Such studies may provide important perspectives regarding the pathophysiology of IIH (especially some specific IIH subtypes) as well as treatment-based clinical approaches to some subgroups of IIH.

\section{Key Messages}

- Obstructive sleep apnea should be kept in mind as an independent etiological factor in IIH, particularly in patients with malignant IIH.

- A patient-specific multimodal approach is needed in these IIH patients for the best clinical responses.

- Distinct mechanisms other than ICP increments may be responsible for prominent papilledema presentations in $\mathrm{IIH}$, which constitutes a crucial issue for clarification in future studies.

\section{References}

1. Ahuja S, Anand D, Dutta TK, Roopesh Kumar VR, Kar SS: Retinal nerve fiber layer thickness analysis in cases of papilledema using optical coherence tomography - a case control study. Clin Neurol Neurosurg 136:95-99, 2015

2. Banta JT, Farris BK: Pseudotumor cerebri and optic nerve sheath decompression. Ophthalmology 107:1907-1912, 2000

3. Berg S: Obstructive sleep apnoea syndrome: current status. Clin Respir J 2:197-201, 2008

4. Brazis PW, Lee AG: Elevated intracranial pressure and pseudotumor cerebri. Curr Opin Ophthalmol 9:27-32, 1998

5. Carvalho B, Hsia J, Capasso R: Surgical therapy of obstructive sleep apnea: a review. Neurotherapeutics 9:710-716, 2012

6. Frisén L: Swelling of the optic nerve head: A staging scheme. J Neurol Neurosurg Psychiatry 45:13-18, 1982

7. Javaheri S, Qureshi Z, Golnik K: Resolution of papilledema associated with OSA treatment. J Clin Sleep Med 7:399400, 2011
8. Jennum P, Børgesen SE: Intracranial pressure and obstructive sleep apnea. Chest 95:279-283, 1989

9. Kalyoussef E, Brooks NO, Quraishi H, Turbin R, Frohman L: Idiopathic intracranial hypertension in a child with obstructive sleep apnea cured by tonsillectomy/adenoidectomy. J Neuroophthalmol 33:413-414, 2013

10. Kidron D, Pomeranz S: Malignant pseudotumor cerebri. Report of two cases. J Neurosurg 71:443-445, 1989

11. Lee AG, Golnik K, Kardon R, Wall M, Eggenberger E, Yedavally S: Sleep apnea and intracranial hypertension in men. Ophthalmology 109:482-485, 2002

12. Mensah A, Milea D, Jensen R, Fledelius H: Persistent visual loss in malignant idiopathic intracranial hypertension. Acta Ophthalmol 87:934-936, 2009

13. Optical Coherence Tomography Substudy Committee: Papilledema outcomes from the optical coherence tomography substudy of the Idiopathic Intracranial Hypertension Treatment Trial. Ophthalmology 122:1939-1945, 1945.e1-1945. e2, 2015

14. Peter L, Jacob M, Krolak-Salmon P, Petitjean T, Bastuji H, Grange JD, et al: Prevalence of papilloedema in patients with sleep apnoea syndrome: a prospective study. J Sleep Res 16:313-318, 2007

15. Sanner BM, Konermann M, Tepel M, Groetz J, Mummenhoff $\mathrm{C}$, Zidek W: Platelet function in patients with obstructive sleep apnoea syndrome. Eur Respir J 16:648-652, 2000

16. Thurtell MJ, Trotti LM, Bixler EO, Rye DB, Bliwise DL, Newman NJ, et al: Obstructive sleep apnea in idiopathic intracranial hypertension: comparison with matched population data. J Neurol 260:1748-1751, 2013

17. Wardly D, Wolford LM, Veerappan V: Idiopathic intracranial hypertension eliminated by counterclockwise maxillomandibular advancement: a case report. Cranio 35:259-267, 2017

18. Wardly DE: Intracranial hypertension associated with obstructive sleep apnea: a discussion of potential etiologic factors. Med Hypotheses 83:792-797, 2014

\section{Disclosures}

The authors report no conflict of interest concerning the materials or methods used in this study or the findings specified in this paper.

\section{Author Contributions}

Conception and design: Onder, Ergun. Acquisition of data: all authors. Analysis and interpretation of data: Onder, Ergun. Drafting the article: Onder. Critically revising the article: Onder. Reviewed submitted version of manuscript: Onder, Ergun. Approved the final version of the manuscript on behalf of all authors: Onder. Administrative/technical/material support: Onder, Kaygisiz, Daltaban. Study supervision: Onder.

\section{Correspondence}

Halil Onder: Yozgat State Hospital, Yozgat, Turkey. halilnder@ yahoo.com. 